\title{
ON DIFFERENTIAL SUBSTITUTIONS FOR EVOLUTION SYSTEMS
}

\author{
S.Ya. STARTSEV
}

\begin{abstract}
For the most known differential substitutions relating scalar evolution equations, the sets of the equations admitting them consist of not finitely many equations but they form families parametrized by an arbitrary function. Some differential substitutions for evolution systems also have a similar property. In the present paper we obtain necessary and sufficient conditions for a differential substitution to be admitted by a family of evolution systems depending on an arbitrary function. We also give explicit formulae for finding the corresponding family of evolution systems in the case when these conditions are satisfied.

As an example, the family of systems admitting a multi-component Cole-Hopf substitution is constructed. We demonstrate that this family contains all linear systems, whose right hand sides contain no terms independent of the derivatives. As a result, we obtain a set of C-integrable systems of arbitrary high order. Another example considered in the paper is a multi-component analogue of the substitution $v=u_{x}+\exp (u)$. We show that this multi-component substitution is also admitted by a family of evolution systems depending on an arbitrary function.
\end{abstract}

Keywords: differential substitutions, evolution systems, C-integrability.

Mathematics Subject Classification: 37K35, 35G50, 37K35

\section{INTRODUCTION}

While studying partial differential equations, an important role is played by differential substitutions. In particular, they allow one both to relate the known integrable equations and to find new equations. The most known substitutions of form

$$
v=P\left(x, u, u_{x}\right)
$$

transform the solutions to the evolution equation

$$
u_{t}=f\left(x, u, u_{1}, \ldots, u_{k}\right), \quad u_{i}:=\frac{\partial^{i} u}{\partial x^{i}},
$$

into the solutions to equations of similar form and these substitutions possess the following property: there exist non-zero operators

$$
S=\sum_{i=0}^{m} \alpha_{i}\left(x, u, u_{1}, \ldots, u_{\ell}\right) D_{x}^{i}, \quad H=\sum_{i=0}^{m+1} \zeta_{i}\left(x, v, v_{1}, \ldots, v_{r}\right) D_{x}^{i},
$$

such that the equation $u_{t}=S\left(\eta\left(x, P, D_{x}(P), \ldots, D_{x}^{\kappa}(P)\right)\right.$ is transformed to the equation $v_{t}=$ $H\left(\eta\left(x, v, v_{1}, \ldots v_{\kappa}\right)\right)$ by substitution (1) for each function $\eta$ depending on finitely many variables. Here $v_{j}:=\partial^{j} v / \partial x^{j}$, while $D_{x}$ stands for the total derivative w.r.t. $x$.

S.Ya. Startsev, On Differential SubStitutions For EVOLUtion Systems.

(C)Startsev S.Ya. 2017.

The work is supported by RSF (grant no. 15-11-20007).

Submitted September 14, $201 \%$. 
The most known example of a differential substitution is the Miura transformation $v=$ $u_{x}-u^{2}$. As it was shown, see, for instance, [1], 2],

$$
S=D_{x}^{2}+2 u D_{x}+2 u_{x}, \quad H=D_{x}^{3}+4 v D_{x}+2 v_{x} .
$$

This is why in what follows, for the brevity, the substitutions with the above properties are called Miura type substitutions.

It was shown in [3] that equation (2) admits the differential substitution $v=P\left(x, u, u_{x}\right)$ if and only if (2) is a symmetry of the hyperbolic equation $u_{x y}=-u_{y} P_{u} / P_{u_{x}}$. If (1) is a Miura type substitution, according [4], the corresponding hyperbolic equation is Darboux integrable. The latter fact allows one to employ the Laplace method of cascade integration both for checking whether (1) is the Miura type transformation and for constructing the corresponding operators $S$ and $H$, see, for instance, [5] or the introduction in work [6].

For systems, that is, in the case $u, f, v$ and $P$ are $n$-dimensional vectors, the differential substitutions are also of interest and they were considered, for instance, in [3], [7]-[11]. However, as it was shown in [12], the Laplace method of cascade integration is, generally speaking, not applicable for the systems. This is why for the system we need some other way of checking whether (1) is a Miura type substitution and of constructing the corresponding operators $S$ and $H$. Such way is proposed in the present work and we show how this works at the examples.

\section{Necessary and sufficient COnditions for Miura type substitutions}

Hereinafter we assume that (2) is a system and (1) is a multi-component substitution of the corresponding dimension. In other words, $u$ and $f$ in (2) and $v$ and $P$ in (1) are $n$-dimensional vectors. Because of this we recall the following standard notations. Given a scalar function $g$ and a vector $z=\left(z^{1}, z^{2}, \ldots, z^{n}\right)^{\top}$, by $g_{z}=\partial g / \partial z$ we denote the row $\left(\partial g / \partial z^{1}, \partial g / \partial z^{2}, \ldots\right.$, $\left.\partial g / \partial z^{n}\right)$. If $g$ is a vector function $\left(g^{1}, g^{2}, \ldots, g^{n}\right)^{\top}$, by $g_{z}$ we denote the matrix with the rows $g_{z}^{1}, \ldots, g_{z}^{n}$. In what follows we assume that the matrix $P_{u_{x}}$ is non-degenerate. It should be noted that not all substitutions (1) for the systems of evolution equations obey this condition, but in the present paper we consider only such substitutions.

Definition 1. We say that system (2) admits differentiable substitution (1) into the system $v_{t}=\hat{f}\left(x, v, v_{1}, \ldots, v_{k}\right)$ if $P_{u_{x}} \neq 0$ and the relation holds ${ }^{1}$

$$
P_{u_{x}} D_{x}(f)+P_{u} f=\hat{f}\left(x, P, D_{x}(P), \ldots, D_{x}^{k}(P)\right) .
$$

We call (1) a Miura type substitution if there exist differential operators of form (3) such that $\alpha_{i}, \zeta_{i}$ are $n$-dimensional vectors, $\alpha_{m} \neq 0$ and for each scalar function $\eta$ depending on finitely many variables the system $u_{t}=S\left(\eta\left(x, P, D_{x}(P), \ldots\right)\right)$ admits substitution (1) into the system $v_{t}=H\left(\eta\left(x, v, v_{1}, \ldots\right)\right)$. In this case the operators $S$ and $H$ are respectively called initial and target substitution operators.

For further arguing, it is convenient to resolve relation (1) w.r.t. $u_{x}$ and to obtain the expression $u_{x}=a(x, u, v)$. Employing the latter identity, we can express each function of $x, u$ and the derivatives of $u$ on $x$ in terms of the variables $x, u, v, v_{i}$. For each scalar function $g$ of these variables, the operator $D_{x}$ is defined by the formula

$$
D_{x}(g)=\frac{\partial g}{\partial x}+\frac{\partial g}{\partial u} a+\frac{\partial g}{\partial v} v_{1}+\sum_{i=1}^{+\infty} \frac{\partial g}{\partial v_{i}} v_{i+1} .
$$

On vectors and matrices, the operator $D_{x}$ acts componentwise. For a shorter writing of formulae, in what follows we assume that the zero power of the operator $D_{x}$, as well as the zero power

\footnotetext{
${ }^{1}$ This relation means that $v=P\left(x, u, u_{x}\right)$ is a solution to the system $v_{t}=\hat{f}$ for each solution of system (2).
} 
of any other operator, is equal to the identity mapping. The symbol $\circ$ denotes the composition of operators.

Theorem 1. If the matrix $P_{u_{x}}$ is non-degenerate, then (1) is a Miura type substitution with an initial operator of order $m$ if and only if there exist $n$-dimensional vectors $\beta_{i}\left(x, v, v_{1}, \ldots, v_{p}\right)$, $i=\overline{0, m+1}$, such that $\beta_{m+1} \neq 0$ and the relation

$$
\sum_{i=0}^{m+1}(-1)^{i}\left(D_{x}-a_{u}\right)^{i}\left(a_{v}\right) \beta_{i}=0
$$

holds, where $P(x, u, a(x, u, v)) \equiv v$.

If (5) is satisfied, then the operators

$$
S=\sum_{i=0}^{m}(-1)^{i}\left(D_{x}-a_{u}\right)^{i}\left(a_{v}\right) \sum_{j=0}^{m-i} D_{x}^{j} \circ \beta_{i+j+1}, \quad H=\sum_{i=0}^{m+1} D_{x} \circ \beta_{i}
$$

are respectively the initial operator (written in terms of the variables $x, u, v, v_{i}$ ) and the target operator of substitution (1).

Proof. Differentiating relation $P(x, u, a(x, u, v)) \equiv v$ w.r.t. $v$ and $u$, we obtain $P_{u_{x}}=\left(a_{v}\right)^{-1}$ and $P_{u}=-\left(a_{v}\right)^{-1} a_{u}$. This is why, after excluding the derivatives of $u$ w.r.t. $x$ by means of the expression $u_{x}=a(x, u, v)$ and its differential consequences, relation (4) becomes

$$
\left(D_{x}-a_{u}\right)\left(f\left(x, u, a, D_{x}(a), \ldots, D_{x}^{k-1}(a)\right)\right)=a_{v} \hat{f}\left(x, v, v_{1}, \ldots, v_{k}\right) .
$$

In the case of Miura type substitution, the latter identity is equivalent to the relation

$$
\left(D_{x}-a_{u}\right) \circ S=a_{v} H
$$

for the initial and target operators (3). Comparing the coefficients at the like powers of $D_{x}$ in the right hand and left hand sides of this relations, we obtain the chain of the identities

$$
\alpha_{m}=a_{v} \zeta_{m+1}, \quad\left(D_{x}-a_{u}\right)\left(\alpha_{i}\right)+\alpha_{i-1}=a_{v} \zeta_{i}, \quad 1 \leqslant i \leqslant m, \quad\left(D_{x}-a_{u}\right)\left(\alpha_{0}\right)=a_{v} \zeta_{0} .
$$

Expressing $\alpha_{i}$ in terms of $\zeta_{j}, j>i$, by means of the first two equations in this chain and substituting then the obtained expression for $\alpha_{0}$ into third equation, we arrive at the relation

$$
\sum_{i=0}^{m+1}(-1)^{i}\left(D_{x}-a_{u}\right)^{i}\left(a_{v} \zeta_{i}\right)=0 .
$$

Identity of form (5) can be obtained easily from (7) by multiple using of the formula

$$
\left(D_{x}-a_{u}\right)\left(a_{v} \zeta\right)=\left(D_{x}-a_{u}\right)\left(a_{v}\right) \zeta+a_{v} D_{x}(\zeta)
$$

Vice versa, if (5) holds, by straightforward check we confirm that the identity $\left(D_{x}-a_{u}\right) \circ S=$ $a_{v} H$ holds for operators (6).

It should be noted that condition (5) and all its consequences obtained by differentiating w.r.t. $u$ is a purely algebraic linear system of equations for finding $\beta_{i}$ and, generally speaking, it is overdetermined. This is why it is rather easy to analyse this condition. Let us demonstrate this by examples. 


\section{Examples of MUlti-COMPOnent Miura type substitutions}

For further discussions, it is convenient to introduce the following notations. For each vector $z=\left(z^{1}, z^{2}, \ldots, z^{n}\right)^{\top}$, by $\langle z\rangle$ we denote the sum of its coordinates, while by $[z]$ we denote the diagonal matrix with the diagonal formed by the coordinates of the vector $z$ :

$$
\langle z\rangle:=\sum_{i=1}^{n} z^{i}, \quad[z]:=\operatorname{diag}\left\{z^{1}, z^{2}, \ldots, z^{n}\right\} .
$$

3.1. Cole-Hopf transformation. A multi-component Cole-Hopf substitution $v=\langle u\rangle^{-1} u_{x}$ was considered, for instance, in works [3], [7]. Let us check whether this is a Miura type substitution.

In order to do this, we write out relation (5) for this substitution with $m=0$. In this case $a=\langle u\rangle v, a_{v}=\langle u\rangle E, a_{u}=[v] C$, where $E$ is the identity matrix and all entries of the matrix $C$ are equal to 1 . It is also easy to see that $D_{x}(\langle u\rangle)=\langle u\rangle\langle v\rangle$. In view of this, in the case of the Cole-Hopf substitution, relation (5) is of the form:

$$
\langle u\rangle \beta_{0}=(\langle u\rangle\langle v\rangle E-\langle u\rangle[v] C) \beta_{1} .
$$

It is easy to see that for each vector $\beta \in \operatorname{ker} C$, i.e., such that $\langle\beta\rangle=0$, the vectors $\beta_{1}=\beta$ and $\beta_{0}=\langle v\rangle \beta$ satisfy this equation. One more solution of $(8)$ is $\beta_{0}=0, \beta_{1}=v$.

Thus, the Cole-Hopf substitution is a Miura type substitution and it has $n$ different initial and target operators (6). They can be considered as two operators with the matrix coefficients $\mathbb{S}=\langle u\rangle B$ and $\mathbb{H}=D_{x} \circ B+\langle v\rangle \tilde{B}$, where the first column of the matrices $B$ and $\tilde{B}$ are respectively the vector $v$ and a zero vector, while other columns of the both matrices coincide and form a basis in $\operatorname{ker} C$. To be definite, as a basis in $\operatorname{ker} C$ we choose the vectors $e_{i}$, whose first coordinate is -1 , the $i$ th coordinate is equal to 1 , and the other coordinates are zero. Under such choice we have

$$
B=\left(\begin{array}{crrrr}
v^{1} & -1 & -1 & \ldots & -1 \\
v^{2} & 1 & 0 & \ldots & 0 \\
v^{3} & 0 & 1 & \ldots & 0 \\
\vdots & \vdots & \vdots & \ddots & \vdots \\
v^{n} & 0 & 0 & \ldots & 1
\end{array}\right)
$$

For each $n$-component vector function $\vec{\eta}$, the transformation $v=\langle u\rangle^{-1} u_{x}$ transforms the system

$$
u_{t}=\mathbb{S}\left(\vec{\eta}\left(x,\langle u\rangle^{-1} u_{x}, D_{x}\left(\langle u\rangle^{-1} u_{x}\right), \ldots\right)\right)
$$

into the system $v_{t}=\mathbb{H}\left(\vec{\eta}\left(x, v, v_{1}, \ldots\right)\right)$.

It was shown in work [7] that each system $u_{t}=\Lambda u_{x x}$, where $\Lambda$ is a constant matrix, admits the substitution $v=\langle u\rangle^{-1} u_{x}$. We are going to generalize this observation by demonstrating that each linear system (2) with $f_{u}=0$ can be represented as (9).

By induction in $i$, it is easy to check that $\langle u\rangle^{-1} u_{i}=\left(D_{x}+\langle P\rangle\right)^{i-1}(P)$, where $i>0$ and $P=\langle u\rangle^{-1} u_{x}$. Indeed, for $i=1$ this identity coincides with the formula $P=\langle u\rangle^{-1} u_{x}$. If it holds for some $i$, this implies that

$$
\frac{u_{i+1}}{\langle u\rangle}=D_{x}\left(\frac{u_{i}}{\langle u\rangle}\right)-D_{x}\left(\frac{1}{\langle u\rangle}\right) u_{i}=\left(D_{x}+\frac{\left\langle u_{x}\right\rangle}{\langle u\rangle}\right)\left(\frac{u_{i}}{\langle u\rangle}\right)=\left(D_{x}+\langle P\rangle\right)^{i}(P) .
$$

In view of this identity we have

$$
A u_{i}=\langle u\rangle B B^{-1} A\left(D_{x}+\langle P\rangle\right)^{i-1}(P)=\mathbb{S}\left(B^{-1} A\left(D_{x}+\langle P\rangle\right)^{i-1}(P)\right)
$$


for each $i>0$ and each matrix $A$. If this matrix can be expressed in terms of $x, P$ and the total derivatives of $P$ w.r.t. $x$, the system $u_{t}=A u_{i}$ can be represented as (9) with $\vec{\eta}=B^{-1} A\left(D_{x}+\langle P\rangle\right)^{i-1}(P)$ and therefore, the Cole-Hopf substitution maps it to the system

$$
v_{t}=\mathbb{H}(\vec{\eta})=D_{x}\left(A\left(D_{x}+\langle v\rangle\right)^{i-1}(v)\right)+\langle v\rangle \tilde{B} B^{-1} A\left(D_{x}+\langle v\rangle\right)^{i-1}(v) .
$$

By straightforward check one can confirm that

$$
B^{-1}=\frac{1}{\langle v\rangle}\left(\begin{array}{ccccc}
1 & 1 & 1 & \ldots & 1 \\
-v^{2} & \langle v\rangle-v^{2} & -v^{2} & \ldots & -v^{2} \\
-v^{3} & -v^{3} & \langle v\rangle-v^{3} & \ldots & -v^{3} \\
\vdots & \vdots & \vdots & \ddots & \vdots \\
-v^{n} & -v^{n} & -v^{n} & \ldots & \langle v\rangle-v^{n}
\end{array}\right) .
$$

It follows from $(\tilde{B}+B-\tilde{B}) B^{-1}=E$ that $\tilde{B} B^{-1}=E+(\tilde{B}-B) B^{-1}$. The first column of the matrix $\tilde{B}-B$ is equal to $-v$, while the other entries of this matrix are zero. This is why $\tilde{B} B^{-1}=E-\langle v\rangle^{-1}[v] C$. Substituting the latter expression into $(10)$, we obtain

$$
v_{t}=\left(D_{x}+\langle v\rangle\right)\left(A\left(D_{x}+\langle v\rangle\right)^{i-1}(v)\right)-\left\langle A\left(D_{x}+\langle v\rangle\right)^{i-1}(v)\right\rangle v .
$$

In particular, this implies that each system of form

$$
v_{t}=\sum_{i=1}^{k}\left(\left(D_{x}+\langle v\rangle\right)\left(A_{i}(t, x)\left(D_{x}+\langle v\rangle\right)^{i-1}(v)\right)-\left\langle A_{i}(t, x)\left(D_{x}+\langle v\rangle\right)^{i-1}(v)\right\rangle v\right),
$$

where $A_{i}$ are matrices of size $n \times n$, is C-integrable since it is obtained from the linear system $u_{t}=\sum_{i=1}^{k} A_{i}(t, x) u_{i}$ by the Cole-Hopf substitution. Here we have added the dependence on $t$ into $\vec{\eta}$, since nothing prevents us from considering substitutions (1) for systems (2) with an explicit dependence on $t$ in the right hand side. The only difference caused by adding $t$ in definition 1 is that $\hat{f}$ in (4) can also depends on $t$. And the dependence on $t$ in $\vec{\eta}$ makes no influence on the defining relation $\left(P_{u_{x}} D_{x}-P_{u}\right) \circ \mathbb{S}=\mathbb{H}$.

3.2. Exponential substitution. . One of the scalar Miura substitutions is the substitution $v=u_{x}+\exp (u)$, see, for instance, [1, 4]. Let us try to construct its multi-component analogue. In order to do this, we denote by $\mathbf{e}^{\mathbf{u}}$ the vector $\left(\exp \left(u^{1}\right), \exp \left(u^{2}\right), \ldots, \exp \left(u^{n}\right)\right)^{\top}$ and let us check whether $v=u_{x}-A \mathbf{e}^{\mathbf{u}}$, where $A$ is a constant $n \times n$ matrix, is a Miura type substitution.

As $m=0$, condition (5) for this substitution is of the form $\beta_{0}+A\left[\mathbf{e}^{\mathbf{u}}\right] \beta_{1}=0$. Differentiating this identity in $u^{i}$, we obtain that the product of $i$ th coordinate of the vector $\beta_{1}$ and of $i$ th column of the matrix $A$ is zero. This is why the substitution $v=u_{x}-A \mathbf{e}^{\mathbf{u}}$ with a non-degenerate matrix $A$, that is, in the general case, does not admit an initial zero order operator.

In the case $m=1$ identity (5) is written as

$$
\beta_{0}+A\left[\mathbf{e}^{\mathbf{u}}\right] \beta_{1}=A\left[\mathbf{e}^{\mathbf{u}}\right]\left(\left[v+A \mathbf{e}^{\mathbf{u}}\right]-A\left[\mathbf{e}^{\mathbf{u}}\right]\right) \beta_{2} .
$$

We denote by $\sharp_{j}^{i}$ the vector, whose coordinates from the $i$ th up to the $j$ th are equal to 1 , while other coordinates are zero. It is easy to see that $\beta_{2}=\sharp_{n}^{1}, \beta_{1}=v$ and $\beta_{0}=0$ is a solution to (11). The corresponding initial and target operators are $S=\sharp_{n}^{1} D_{x}+u_{x}, H=\sharp_{n}^{1} D_{x}^{2}+v D_{x}+v_{x}$. Thus, $v=u_{x}-A \mathbf{e}^{\mathbf{u}}$ is a Miura type substitution for each constants matrix $A$. For a special matrix $A$ this substitution can admit additional initial and target operators. Apart of the matrix $A$ with one or more zero columns discussed in the previous paragraph, additional initial and target operators can also arise under the condition $\operatorname{det}(A) \neq 0$.

For instance, if the matrix $A$ is block diagonal with $i$ th block located in the rows and columns from $p_{i}$ th to $q_{i}$ th, then $\beta_{2}=\sharp_{q_{i}}^{p_{i}}, \beta_{1}=[v] \sharp_{q_{i}}^{p_{i}}$ and $\beta_{0}=0$ are also solutions of (11). In particular, if the matrix $A$ is diagonal, that is, the size of all blocks is 1 , the corresponding set of initial and 
target operators can be written as $\mathbb{S}=E D_{x}+\left[u_{x}\right], \mathbb{H}=E D_{x}^{2}+[v] D_{x}+\left[v_{x}\right]$. We can not also exclude that additional initial and target operators can be found while analysing relation (5) with $m>1$.

\section{BIBLIOGRAPHY}

1. S.V. Khabirov. Bäcklund transformations of evolution equations // Preprint. Bashkir Branch of AS USSR, Ufa. (1984). (in Russian).

2. S.Yu. Sakovich. On the polynomial Miura transformation // Phys. Lett. A. 146:1,2, 32-34 (1990).

3. V.V. Sokolov. On the symmetries of evolution equations // Uspekhi Matem. Nauk. 43:5(263), 133-163 (1988). [Russ. Math. Surv. 43:5, 165-204 (1988).]

4. S.Ya. Startsev. Differential substitutions of the Miura transformation type // Teor. Matem. Fiz. 116:3, 336-348 (1998). [Theoret. Math. Phys. 116:3, 1001-1010 (1998).]

5. F.G. Tricomi. Lectures on partial differential equations. Inostr. Liter., Moscow (1957). [Lezioni sulle equazioni a derivate parziali. Editrice Gheroni, Torino (1954). (in Italian).]

6. S.Ya. Startsev. Cascade method of Laplace integration for linear hyperbolic systems of equations // Matem. Zametki. 83:1, 107-118 (2008). [Math. Notes. 83:1, 97-106 (2008).]

7. S.I. Svinolupov, V.V. Sokolov. Factorization of evolution equations // Uspekhi Matem. Nauk. 47:3(285), 115-146 (1992). [Russ. Math. Surv. 47:3, 127-162 (1992).]

8. D.K. Demskoi. One Class of Liouville-type systems // Teor. Matem. Fiz. 141:2, 208-227 (2004). [Theor. Math. Phys. 141:2, 1509-1527 (2004).]

9. A.V. Kiselev. Algebraic properties of Gardner's deformations for integrable systems // Teor. Matem. Fiz. 152:1, 101-117 (2007). [Theor. and Math. Phys. 152:1, 963-976 (2007).]

10. M.Yu. Balakhnev. First-order differential substitutions for equations integrable on $\mathbb{S}^{n} / /$ Matem. Zametki. 89:2, 178-189 (2011). [Math. Notes. 89:2, 184-193 (2011).]

11. M. Yu. Balakhnev. Differential substitutions for vectorial generalizations of the $m K d V$ equation // Matem. Zametki. 98:2, 173-179 (2015). [Math. Notes. 98:2, 204-209 (2015).]

12. A.V. Zhiber, S.Ya. Startsev. Integrals, solutions, and existence Problems for Laplace transformations of linear hyperbolic systems // Matem. Zametki. 74:6, 848-857 (2003). [Math. Notes. 74:6, 803-811 (2003).]

Sergey Yakovlevich Startsev,

Institute of Mathematics,

Ufa Scientific Center,

Russian Academy of Sciences,

Chernyshevskii str. 112,

450077, Ufa, Russia

E-mail: startsev@anrb.ru 